University of Nebraska - Lincoln

DigitalCommons@University of Nebraska - Lincoln

June 2000

\title{
Photoionization and photofragmentation of gaseous toluene using 80-fs, 800-nm laser pulses
}

\author{
A. M. Müller \\ Max-Planck-Institut für Quantenoptik, D-85741 Garching, Germany \\ Cornelis J. Uiterwaal \\ University of Nebraska - Lincoln, cuiterwaal2@unl.edu \\ B. Witzel \\ Foundation for Research and Technology-Hellas, Institute of Electronic Structure \& Laser, Laser and \\ Applications Division, P.O. Box 1527, GR-71110 Heraklion, Greece \\ J. Wanner \\ Max-Planck-Institut für Quantenoptik, D-85741 Garching, Germany \\ K.-L. Kompa \\ Max-Planck-Institut für Quantenoptik, D-85741 Garching, Germany
}

Follow this and additional works at: https://digitalcommons.unl.edu/physicsuiterwaal

Part of the Physics Commons

Müller, A. M.; Uiterwaal, Cornelis J.; Witzel, B.; Wanner, J.; and Kompa, K.-L., "Photoionization and photofragmentation of gaseous toluene using $80-\mathrm{fs}, 800-\mathrm{nm}$ laser pulses" (2000). C.J.G.J. Uiterwaal Publications. 6.

https://digitalcommons.unl.edu/physicsuiterwaal/6

This Article is brought to you for free and open access by the Research Papers in Physics and Astronomy at DigitalCommons@University of Nebraska - Lincoln. It has been accepted for inclusion in C.J.G.J. Uiterwaal Publications by an authorized administrator of DigitalCommons@University of Nebraska - Lincoln. 


\title{
Photoionization and photofragmentation of gaseous toluene using 80-fs, 800-nm laser pulses
}

\author{
A. M. Müller and C. J. G. J. Uiterwaal ${ }^{\text {a) }}$ \\ Max-Planck-Institut für Quantenoptik, P.O. Box 1327, D-85741 Garching, Germany \\ B. Witzel \\ Foundation for Research and Technology-Hellas, Institute of Electronic Structure \& Laser, \\ Laser and Applications Division, P.O. Box 1527, GR-71110 Heraklion, Greece \\ J. Wanner and K.-L. Kompa \\ Max-Planck-Institut für Quantenoptik, P.O. Box 1327, D-85741 Garching, Germany
}

(Received 4 January 2000; accepted 6 March 2000)

\begin{abstract}
This paper presents ion yields resulting from multiphoton ionization and fragmentation of gaseous toluene $\left(\mathrm{C}_{7} \mathrm{H}_{8}\right)$ in the focus of an $80 \mathrm{fs}$ Ti:sapphire laser beam $(\lambda=800 \mathrm{~nm})$ with a sufficiently small $B$-integral [Siegman, Lasers (University Science Books, Mill Valley, CA, 1986)]. The peak intensity was varied between $1.9 \times 10^{13}$ and $2.8 \times 10^{14} \mathrm{~W} \mathrm{~cm}^{-2}$, and both linear and circular polarization were used. Over the whole range of intensities studied, only the singly charged parent ion and its fragment, $\mathrm{C}_{7} \mathrm{H}_{7}^{+}$, are found. Although the Keldysh adiabaticity parameter equals 0.86 for the saturation intensity of $\sim 1 \times 10^{14} \mathrm{~W} \mathrm{~cm}^{-2}$, there is no indication of tunneling. The parent ion yield is found to be effectively proportional to the sixth power of the peak intensity. This is shown to be in good agreement with a multiple lowest-order perturbation multiphoton ionization model which takes into account successive channel closing for increasing peak intensities and orders up to 11 inclusive. On the assumption that the excess energy acquired by the toluene cation as a result of the interaction with the electromagnetic field is of the order of the ponderomotive energy for the intensity prevailing at the moment of the ionization, the internal energy distribution of the toluene cations created that is brought about by this multiple-order multiphoton ionization model is calculated. This internal energy distribution is in perfect agreement with the measured $\mathrm{C}_{7} \mathrm{H}_{7}^{+}$yield, if the rate-energy curve for the fragmentation of excited toluene cations as given by Golovin et al. [Sov. J. Chem. Phys. 2, 632 (1985)] is moderately reduced by a factor of 4.5. () 2000 American Institute of Physics. [S0021-9606(00)00121-5]
\end{abstract}

\section{INTRODUCTION}

The photodynamics of molecules under the influence of short $(<100 \mathrm{fs})$, strong laser pulses is now becoming a topic of wide interest. For intensities beyond $1.3 \times 10^{13} \mathrm{~W} \mathrm{~cm}^{-2}$, the electric field produced by a laser exceeds the value 1 $\mathrm{V} \AA^{-1}$. The behavior of molecules exposed to such strong fields, which are comparable in strength to the electrostatic fields that are responsible for the stability of molecules, is more complex than one might expect. For instance, it was shown very recently ${ }^{1-3}$ that stable, multiply charged parent ions of medium-size aromatic molecules are created by intense $800-\mathrm{nm}$, sub-90-fs laser pulses, and that photofragmentation plays a very minor role under these conditions. This "atomiclike" behavior, which favors "soft ionization" leading predominantly to parent ions and is therefore sometimes called femtosecond laser mass spectrometry (FLMS), is surprising, because at the energies required to create higher charge states several fragmentation channels are known to be easily accessible. Results of similar photoionization and photofragmentation experiments on medium-size aromatic

\footnotetext{
a) Author to whom correspondence should be addressed. Electronic mail: Kees.Uiterwaal@mpq.mpg.de
}

and nonaromatic molecules with various degrees of bond saturation were also published recently. ${ }^{4-7}$ Experiments of this kind strongly contribute to the data-gathering phase, which must be the first step taken whenever a new field of research is being explored, as is the case with intense, ultrashort laser field molecular photoionization and photofragmentation. A logical next step would be a careful inspection of the data collected to identify and resolve possible controversies. This is the approach adopted here to study the photoionization and photofragmentation of the toluene molecule. Recently, the photodynamics of this molecule were investigated by several groups, ${ }^{1-4,6}$ who do not agree, however, on a common ionization/fragmentation scenario. We claim that these discrepancies are largely due to unwanted contributions from nonlinear effects in the laser beam (as expressed by, for example, the so-called $B$-integral ${ }^{8}$ ). Therefore, in the present "case study" of toluene, the experimental conditions under which the ionization/fragmentation data were obtained are carefully and extensively defined and discussed. Especially in high-intensity laser physics, a precise characterization of laser beams is a conditio sine qua non for a proper understanding of experimental work. A further unresolved question seems to be the exact nature of the ionization process of 
molecules in FLMS. For the case of an atom with an ionization energy (IE), the often-cited and well-known Keldysh adiabaticity parameter $\gamma,{ }^{9}$ is defined as

$$
\gamma=\sqrt{\frac{I E}{2 U_{p}}},
$$

where $U_{p}$ is the so-called ponderomotive or quiver energy, makes a rough subdivision of ionization processes into multiphoton ionization (MPI) processes (for which $\gamma \gg 1$ ) and tunneling processes (for which $\gamma \ll 1$ ). Useful as this is as a starting point, this subdivision is not so sharp as one might wish it to be, and ionization processes will often be a mixture of these two extremes. Furthermore, the Keldysh parameter was derived for structureless particles (atoms), and although often mentioned in relation to molecular studies it does not take into account typical features of molecules such as their extended size in relation to atomic systems. At least one group has introduced some kind of molecular Keldysh parameter ${ }^{5}$ and successfully applied it to a specific class of molecules. Probably, however, the complexity of molecular systems and their photodynamics are so comprehensive that a "general" description must involve more than just a single classification parameter. Aiming to identify the ionization process of toluene, we followed its ion yields over several orders of magnitude as a function of laser peak intensity in the range between $1.9 \times 10^{13} \mathrm{~W} \mathrm{~cm}^{-2}(\gamma \approx 2)$ and 2.8 $\times 10^{14} \mathrm{~W} \mathrm{~cm}^{-2}(\gamma \approx 0.5)$, and our results are explained in the framework of a multiple-order MPI model. The fragmentation of toluene, which is limited to just a hydrogen atom loss process up to the highest peak intensity of 2.8 $\times 10^{14} \mathrm{~W} \mathrm{~cm}^{-2}$ used here, can be fully explained by directly connecting this multiple-order MPI mechanism to the energy content of the ion created. This paper is organized as follows: Sec. II describes the experimental setup; in Sec. III we present and discuss the results of toluene photoionization together with our calculations; Sec. IV is devoted to conclusions.

\section{EXPERIMENT}

A commercial Ti:sapphire laser system (SpectraPhysics) was used for the present work. In a chirped-pulse amplification scheme, pulses from a Ti:sapphire oscillator (Tsunami, wavelength $795 \mathrm{~nm}$, power $900 \mathrm{~mW}$, repetition rate $82 \mathrm{MHz}$, pulse duration $65 \mathrm{fs}$ ) pumped by a frequencydoubled Nd: $\mathrm{YVO}_{4}$ laser (Millennia-V, $532 \mathrm{~nm}, 4.90 \mathrm{~W}$, cw) are fed into a regenerative amplifier (Super-Spitfire). The amplifier is pumped by a frequency-doubled, Q-switched Nd:YLF laser (Super-Merlin, $527 \mathrm{~nm}, 23 \mathrm{~W}, 1 \mathrm{kHz}$ ). The laser system finally delivers pulses at a repetition rate of 1 $\mathrm{kHz}$ with an energy of $2.0 \mathrm{~mJ} /$ pulse (shot-to-shot stability $\pm 4 \%$ ). Pulse energies were measured with a power meter (Laser Probe power meter Rm 6600 universal radiometer). A commercial autocorrelator (APE Pulsecheck) was used to measure the temporal pulse width directly after the compression grating (typically $\sim 70 \mathrm{fs}$ ). The output wavelength was $800 \mathrm{~nm}$, as determined with a fiber spectrometer (Ocean Optics SD2000).
From measurements of the fractional power transfer through centered circular apertures of various diameters it is concluded that the spatial profile of the unfocused laser beam is Gaussian and has a FWHM diameter of $d_{\mathrm{FWHM}}=(5.37$ $\pm 0.02) \mathrm{mm}$. Furthermore, autocorrelation measurements using a second autocorrelator (APE Pulsecheck 75050 ) show that the temporal profile of the pulses right before the focusing lens (after ten reflections from dielectric mirrors) also has a Gaussian shape with a FWHM duration of $t_{\mathrm{FWHM}}=(81$ \pm 1 ) fs. Therefore, the spatio-temporal variation of the intensity in the unfocused beam is given by

$$
I(r, t)=I_{0} \exp \left(-\left(r / r_{0}\right)^{2}\right) \exp \left(-\left(t / t_{0}\right)^{2}\right)
$$

with $r_{0}=(3.23 \pm 0.01) \mathrm{mm}$ and $t_{0}=(48.6 \pm 0.6) \mathrm{fs}$. For the highest pulse energy of $1.2 \mathrm{~mJ}$ used in our experiments, the peak intensity in the unfocused beam is then $I_{0}=4.2$ $\times 10^{10} \mathrm{~W} \mathrm{~cm}^{-2}$.

To focus the laser beam, a plano-convex uncoated BK-7 lens with a nominal focal length of $f=500 \mathrm{~mm}$ was used. Measurements of the spatial beam profile at focus were made by means of a CCD camera (Spiricon Pulnix TM-6CN). This profile is again Gaussian and has a typical FWHM diameter of $\sim 60 \mu \mathrm{m}$, in agreement with calculations. Attenuation of the unfocused beam was performed by means of a rotatable variable-sector filter disk. The different sectors of this $2 \mathrm{~mm}$ thick quartz disk have metal coatings of various thicknesses, which allows the intensity to be reduced in steps of $\sim 63 \%$ (optical density 0.2 ). To bridge the gap between these steps, an additional $1.1 \mathrm{~mm}$ thick Corning 7059 metal-coated neutral density filter was used with a $\sim 79 \%$ transmission (optical density 0.1 ). The entrance window of the vacuum system is a $2 \mathrm{~mm}$ thick $\mathrm{CaF}_{2}$ optical flat positioned $\sim 280 \mathrm{~mm}$ from the focus. An important aspect of our setup is the possible role of nonlinear effects, such as self-focusing or self-phase modulation, that may be induced by nonlinear behavior of the optical materials $\mathrm{BK}-7$ and $\mathrm{CaF}_{2}$ due to the rather high unfocused intensity of the laser beam. However, even for the highest unfocused peak intensity $I_{0}=4.2 \times 10^{10} \mathrm{~W} \mathrm{~cm}^{-2}$ used here the cumulative $B$-integral ${ }^{8}$ in our setup for air plus optics amounts to at most 1.7. Since this is way below the widely accepted critical limit of 3 to 5 (see Ref. 8), nonlinear effects are not expected to have any influence. Measurements of the temporal autocorrelation function and the frequency spectrum of the pulse after its passage through the entrance window of the vacuum system showed that the laser pulse does remain essentially unaltered, which verifies our expectations.

A simple time-of-flight (TOF) arrangement based on the Wiley-McLaren principle ${ }^{10}$ was used for cation mass analysis. The spectrometer axis is perpendicular to the propagation direction of the laser beam. After their creation in the focus of the laser beam, the ions pass through a two-stage acceleration sector (first stage, length $10 \mathrm{~mm}$, field strength 241 $\mathrm{V} \mathrm{cm}^{-1}$; second stage, length $20 \mathrm{~mm}$, field strength 996 $\mathrm{V} \mathrm{cm}^{-1}$ ). The last electrode of the second acceleration stage consists of a solid plate in which a rectangular slit is present with dimensions $10.0 \mathrm{~mm} \times 1.2 \mathrm{~mm}$, its longer dimension being parallel to the propagation direction of the laser beam. After passing through this slit, the ions drift through a 760 

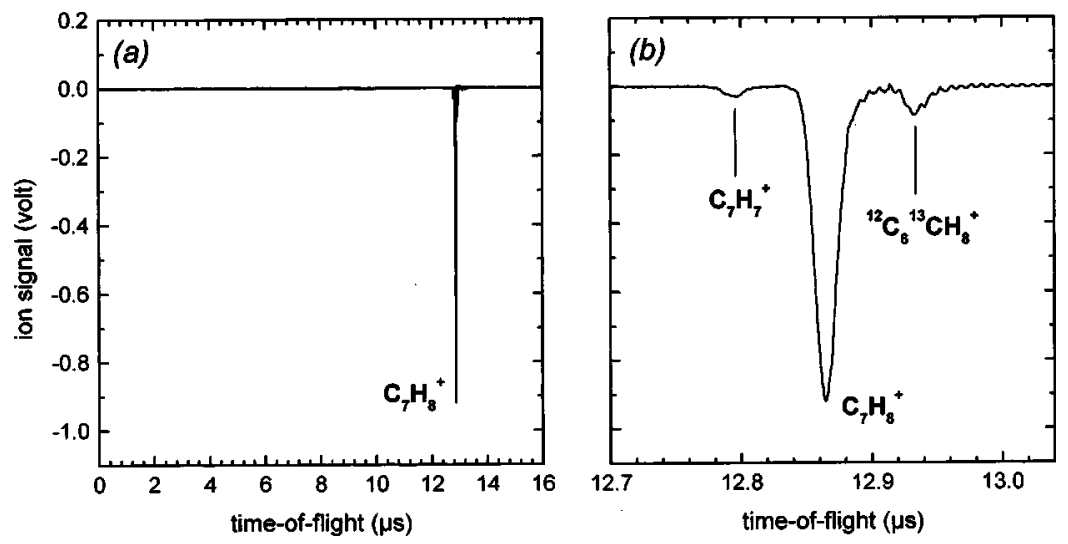

FIG. 1. TOF spectrum of toluene recorded with 800$\mathrm{nm}, 80$-fs, linearly polarized laser pulses for a peak intensity of $2.0 \times 10^{14} \mathrm{~W} \mathrm{~cm}^{-2}$. (a) Complete spectrum; (b) expanded view of the same spectrum. The spectrum is dominated by the parent ion, $\mathrm{C}_{7} \mathrm{H}_{8}^{+}(M=92)$, accompanied by its heavier isotopic variant, ${ }^{12} \mathrm{C}_{6}{ }^{13} \mathrm{CH}_{8}^{+}(M$ =93). Only one other mass peak is found, corresponding to the fragment ion, $\mathrm{C}_{7} \mathrm{H}_{7}^{+}(M=91)$, which is created in limited quantities.

$\mathrm{mm}$ long field-free region. After leaving the drift region, they are accelerated to a kinetic energy of $\sim 4.4 \mathrm{keV}$ and then impinge on the front face of a double-stage multichannelplate (MCP) assembly operated at typically $2 \mathrm{kV}$ which is used for ion detection. The resolution of the TOF spectrometer amounts to $M / \Delta M \sim 200$. Ion yields were measured with both linear and circular polarization being used. To change the polarization of the beam, a zero-order air-spaced quarter-wave plate was used (Halle, RZQ 4.15), with its fast axis either parallel to the linear polarization of the laser beam or at $45^{\circ}$. The linear polarization was perpendicular to the spectrometer axis. This direction was chosen because it allows us to use one and the same quarter-wave plate to change the polarization. Additional experiments in which a half-wave plate was used to make the linear polarization parallel to the spectrometer axis showed that the results for parallel and perpendicular linear polarization are essentially identical. To align the focus to the axis of the spectrometer, the yield of $\mathrm{C}_{7} \mathrm{H}_{8}^{+}$(this is by far the dominating peak in the spectrum) was maximized by moving the position of the lens.

Gaseous toluene was admitted to the vacuum chamber by means of a variable leak connected to a flask containing liquid toluene and its saturated vapor at room temperature. Toluene with purity $>99.9 \%$ (Merck Uvasol $^{\circledR}$ ) was used after being subjected to a few pump-freeze-thaw cycles using liquid nitrogen. Depending on the intensity, toluene pressures between $1 \times 10^{-7} \mathrm{mbar}$ and $2 \times 10^{-5}$ mbar were used. These pressures were measured with an ionization gauge and multiplied by a correction factor accounting for the increased sensitivity of the gauge for toluene with respect to molecular nitrogen. The rest gas pressure is estimated to be typically $2 \times 10^{-8}$ mbar. During the measurements, the pressure in the drift sector of the mass spectrometer, which is separated from the main (interaction) chamber by the slit and is differentially pumped, was below $2 \times 10^{-7}$ mbar. To record the ion TOF spectra, the MCP signal was fed into a preamplifier (Stanford Research SR240). By using a digital oscilloscope (LeCroy LC564A, $1 \mathrm{GHz}$ ) the resulting signal was averaged over one thousand laser pulses and then stored.

\section{RESULTS AND DISCUSSION}

A typical TOF spectrum recorded with linearly polarized radiation at a peak intensity of $2.0 \times 10^{14} \mathrm{~W} \mathrm{~cm}^{-2}$ is shown in
Fig. 1. The spectrum is dominated by the parent ion $\mathrm{C}_{7} \mathrm{H}_{8}^{+}(M=92)$. The only fragment produced in observable quantities is $\mathrm{C}_{7} \mathrm{H}_{7}^{+}(M=91)$. The peak at $M=93$ is due to ${ }^{12} \mathrm{C}_{6}{ }^{13} \mathrm{CH}_{8}^{+}$. For the whole intensity range studied here (between $1.9 \times 10^{13}$ and $2.8 \times 10^{14} \mathrm{~W} \mathrm{~cm}^{-2}$ ), and for both linear and circular polarization, these are the only peaks that were observed.

In an initial phase of the experiment, we recorded one TOF spectrum with an $f=1000 \mathrm{~mm}$ plano-convex lens for a peak intensity of $6.4 \times 10^{13} \mathrm{~W} \mathrm{~cm}^{-2}$. With this lens some fragmentation products that were absent with the $f$ $=500 \mathrm{~mm}$ lens appear, such as $\mathrm{C}_{3} \mathrm{H}_{i}^{+}(i=0, \ldots, 5), \mathrm{C}_{4} \mathrm{H}_{i}^{+}(i$ $=0, \ldots, 5), \mathrm{C}_{5} \mathrm{H}_{i}^{+}(i=3,5,6), \mathrm{C}_{6} \mathrm{H}_{5}^{+}$, and $\mathrm{C}_{7} \mathrm{H}_{i}^{+}(i=5,6)$, but, more surprisingly, at the same time doubly charged ions such as $\mathrm{C}_{7} \mathrm{H}_{i}^{2+}(i=6,7,8)$ appear that are also definitely absent in all our other TOF spectra, some of which were even recorded for higher intensities! On the other hand, the use of a lens with a shorter focal length of $300 \mathrm{~mm}$ leads to the same mass spectra as obtained with the $500 \mathrm{~mm}$ lens. To understand this result, it must be noted that the use of the $f$ $=1000 \mathrm{~mm}$ lens exposes the air between the lens and the $\mathrm{CaF}_{2}$ entrance window of the vacuum system plus the entrance window itself to intensities of up to 5.5 $\times 10^{11} \mathrm{~W} \mathrm{~cm}^{-2}$, and as a result the $B$-integral grows to 2.9 . This suggests that the laser beam is strongly distorted by nonlinear effects, and all of the striking new features that we observe in the TOF spectrum must be ascribed to this. It is known ${ }^{11}$ that self-focusing effects trigger continuum generation. Most probably, therefore, the high value of the $B$-integral indicates that we have created conditions under which the effects of continuum generation become noticeable. Indeed, measurements of the frequency spectrum show that it changes considerably; a marked broadening around $800 \mathrm{~nm}$ is observed, and a whole group of additional frequencies with wavelengths between 400 and $650 \mathrm{~nm}$ emerges as well. The latter profound changes in the highfrequency side are most probably a manifestation of the onset of continuum generation. Therefore, a comparison of the TOF spectrum obtained with the $f=1000 \mathrm{~mm}$ lens with the TOF spectra obtained with the $f=500 \mathrm{~mm}$ lens can only be done with utmost reservation.

In a very recent publication, ${ }^{4}$ Castillejo et al. present TOF spectra resulting from ionization and fragmentation of toluene and a small number of other aromatic and sigma- 


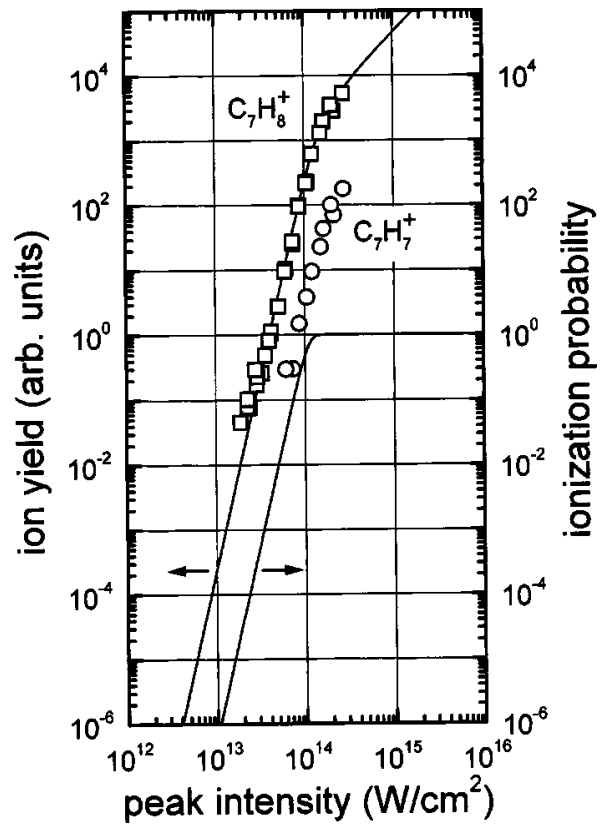

FIG. 2. Ion yields as a function of peak intensity for $800-\mathrm{nm}, 80$-fs, linearly polarized laser pulses irradiating gaseous toluene. Squares, parent ion, $\mathrm{C}_{7} \mathrm{H}_{8}^{+}$; circles, fragment ion, $\mathrm{C}_{7} \mathrm{H}_{7}^{+}$. The solid line passing through the parent ion data (left ordinate axis) was obtained by volume-integrating the ionization probability for a six-photon process (lower solid curve, right ordinate axis).

bonded hydrocarbons obtained under similar conditions (800 $\mathrm{nm}, 50 \mathrm{fs}$ ) for intensities up to $2.5 \times 10^{14} \mathrm{~W} \mathrm{~cm}^{-2}$. Surprisingly, they observe fragmentation products such as $\mathrm{C}_{2} \mathrm{H}_{n}^{+}$ and $\mathrm{C}_{3} \mathrm{H}_{n}^{+}$already at $3.1 \times 10^{13} \mathrm{~W} \mathrm{~cm}^{-2}$, and find the doubly charged parent ion for intensities above $\sim 10^{14} \mathrm{~W} \mathrm{~cm}^{-2}$. Neither observation is in agreement with our results, which show no doubly charged ions, and where the only fragmentation product is $\mathrm{C}_{7} \mathrm{H}_{7}^{+}$in limited amounts. The latter photodynamics of the toluene molecule is in qualitative agreement with the results of Refs. 1-3. It must be noted, however, that the TOF spectra of Castillejo et al. are in fact qualitatively very similar to the spectrum we measure when nonlinear effects distort our beam properties as just described. Therefore, we believe that there is a considerable degree of nonlinear distortion in the laser beam of Castillejo et al. One reason for this could be that their attenuation method makes use of Glan polarizing prisms. This has the obvious disadvantage that the laser beam has to travel through several centimeters of optical material, probably making their $B$-integral too large.

In our TOF spectra recorded with the $f=500 \mathrm{~mm}$ lens, in which case the beam is not subjected to distortions caused by nonlinear effects, the only species found in measurable quantities are $\mathrm{C}_{7} \mathrm{H}_{8}^{+}$and $\mathrm{C}_{7} \mathrm{H}_{7}^{+}$. The yields of these two species as a function of the peak intensity in the pulse are shown in Fig. 2 (for linear polarization) in double-logarithmic representation. The peak intensities were calculated from measured values of the pulse energy, pulse duration, and spatial width of the focus. The ion yields were determined as integrals of the corresponding mass peaks. Because the yield of $\mathrm{C}_{7} \mathrm{H}_{7}^{+}$is only a few percent of the parent ion yield, there was no need to correct the ion yields for the presence of the heavy carbon isotope ${ }^{13} \mathrm{C}$ (natural abundance $1.1 \%$ ) in the toluene molecule, the ratio between ${ }^{12} \mathrm{C}_{6}{ }^{13} \mathrm{CH}_{n}^{+} /{ }^{12} \mathrm{C}_{7} \mathrm{H}_{n}^{+}$being equal to 7.8\%. Even for the highest intensities, where the parent ion yield starts deviating from a straight line, this ratio is found for the parent ion and its heavier satellite, indicating that the bending is due to saturation of the ionization process, and that no saturation of the experimental detection setup takes place which could conceivably cause a similar kind of bending.

As Fig. 2 shows, the nonsaturated $\mathrm{C}_{7} \mathrm{H}_{8}^{+}$data points are all on a straight line; a linear fit gives $5.94 \pm 0.10$ for the slope of this line. Evidently, the parent ion yield increases proportionally to the sixth power of the intensity. This would be in agreement with lowest-order perturbation theory behavior for minimal field intensity; for the $1.55 \mathrm{eV}$ photon energy used here, six photons would be the minimum number required to ionize the toluene molecule, which has an ionization energy of $I E=(8.828 \pm 0.001) \mathrm{eV} .{ }^{12}$ As already mentioned, the deviation from the straight line of the data points obtained for the highest intensities is due to saturation of the ionization process, i.e., to depletion of the ensemble of neutral toluene molecules. For an intensity-independent generalized six-photon cross section $\sigma_{(6)}\left(\right.$ in $\mathrm{cm}^{12} \mathrm{~s}^{5}$ ), the ionization probability depends on the peak intensity $I_{0}$ according to

$$
P\left(I_{0}\right)=1-\exp \left[-\sigma_{(6)}\left(\frac{I_{0}}{\hbar \omega}\right)^{6} t_{\mathrm{eff}}\right]
$$

where the effective pulse duration has been introduced, which for our Gaussian pulse shape can be written as $t_{\text {eff }}$ $=\int_{-\infty}^{\infty} \exp \left(-6\left(t / t_{0}\right)^{2}\right) d t$ and equals $\sqrt{(\pi / 6)} t_{0}$ or 35.2 fs. With the saturation intensity $I_{\text {sat }}$ defined as the intensity for which the ionization probability has a value of $1-e^{-1} \approx 0.632$, the effective six-photon cross section can be written as

$$
\sigma_{(6)}=\left(\frac{\hbar \omega}{I_{\text {sat }}}\right)^{6} t_{\text {eff }}^{-1}
$$

The solid line in Fig. 2 represents a best fit to the experimental data points based on Eq. (3). In this fit, we have taken into account the volume effect [see Eq. (9) below], assuming the focus to have a Gaussian spatial profile, which has been experimentally verified by imaging techniques (see above). From the fit, a saturation intensity of $I_{\mathrm{sat}}=1.0$ $\times 10^{14} \mathrm{~W} \mathrm{~cm}^{-2}$ and a corresponding generalized six-photon cross section of $\sigma_{(6)}=5.9 \times 10^{-183} \mathrm{~cm}^{12} \mathrm{~s}^{5}$ are found. The latter value can be assessed by comparing it with a scaling law value. ${ }^{13}$ Taking the cubic root of the polarizability (12.3 $\AA^{3}$ ) as a rough measure of the size of the toluene molecule, we find a scaling law value of $\sigma_{(6)} \approx 2.0 \times 10^{-177} \mathrm{~cm}^{12} \mathrm{~s}^{5}$. The experimental value is more than five orders of magnitude smaller than this scaling law value, but it must be kept in mind that the scaling law was derived for structureless atoms, and not for molecules. Furthermore, it must be noted that a six-photon generalized cross section is the square of an effective six-photon dipole matrix element; a difference of five orders of magnitude is thus equivalent to a difference of only $\left(10^{5}\right)^{1 / 12}$, or less than a factor of three, in a dipole matrix element of a single photon transition. 
Smith et l. $^{2}$ have recorded ion mass spectra for benzene, toluene, and naphthalene using both 375-nm, 90-fs and 750-nm, 50-fs pulses (both linearly polarized). As we noted, the toluene parent ion yield that these authors recorded for $750 \mathrm{~nm}$ between $\sim 2.4 \times 10^{13} \mathrm{~W} \mathrm{~cm}^{-2}$ and $\sim 3.5$ $\times 10^{14} \mathrm{~W} \mathrm{~cm}^{-2}$ can again be fitted to a curve based on Eq. (3) assuming a Gaussian focus; this procedure then gives a saturation intensity of $3.2 \times 10^{13} \mathrm{~W} \mathrm{~cm}^{-2}$. Smith et al. note that their $375 \mathrm{~nm}$ curves are less steep than their $750 \mathrm{~nm}$ curves, and interpret this as indicating a MPI process, although they speculate on the possibility of tunneling ionization. Unfortunately, Smith et al. recorded their ionization curves largely in the saturation regime, where the ion yield has the well-known volume-determined $I^{3 / 2}$ dependence on the peak intensity, and only a few points recorded at their lowest intensities are available that could give information on the ionization process. As a result, not much can be said on the order of the ionization process; although their data are not at variance with an order of six, we noticed that a somewhat different order (e.g., five) could just as well be fitted to their data. In the present work, the increase of the ion yield was followed over almost four orders of magnitude before saturation set in. For a wavelength of $750 \mathrm{~nm}$, Smith et al. also observe multiply charged parent ions for intensities above $\sim 5 \times 10^{13} \mathrm{~W} \mathrm{~cm}^{-2}$, whereas our $800 \mathrm{~nm}$ spectra do not show multiple ionization up to the highest peak intensity of $2.8 \times 10^{14} \mathrm{~W} \mathrm{~cm}^{-2}$.

In another publication of the Glasgow group, ${ }^{3}$ it is reported that the molecules 1,3-butadiene, carbon disulphide, deuterated benzene, toluene, and naphthalene are multiply ionized when irradiated with laser pulses with a duration below $90 \mathrm{fs}$, a wavelength between 750 and $790 \mathrm{~nm}$, and an intensity of up to $10^{15} \mathrm{~W} \mathrm{~cm}^{-2}$; fragmentation does not occur to any great degree. Doubly and triply charged parent ions are observed for all these molecules (for toluene, the doubly charged parent ion is the highest charge state observed). This behavior is the result of a sequential ionization process, and the authors refer to the sequential mechanism proposed by Lambropoulos $^{14}$ for multiple ionization of atoms under similar circumstances. The $1+$ level is believed to be due to MPI processes, whereas the higher charge states are ascribed to tunneling and/or over-the-barrier mechanisms. The very modest amount of fragmentation, which essentially consists of hydrogen loss processes, and the notion of a MPI mechanism that creates the $1+$ species are in agreement with the present work. The data presented in Ref. 3 show that the tendency towards multiple ionization is least for toluene; even for $2.9 \times 10^{15} \mathrm{~W} \mathrm{~cm}^{-2}$, the height of the $2+$ peak is less than $20 \%$ of the $1+$ peak and the $3+$ peak is absent, whereas for all other molecules studied by these authors a larger relative $2+$ signal and a clear $3+$ peak are already found for lower intensities. In agreement with this finding, we do not observe any $2+$ signal for toluene for our highest peak intensity of $2.8 \times 10^{14} \mathrm{~W} \mathrm{~cm}^{-2}$.

DeWitt et al. ${ }^{6}$ have the photoionization and photodissociation of benzene, toluene, ethylbenzene, and $n$-propylbenzene using 780-nm, 170-fs radiation in the intensity range between $1.0 \times 10^{13}$ and $3.8 \times 10^{13} \mathrm{~W} \mathrm{~cm}^{-2}$, finding limited dissociation $(7 \%)$ for the highest intensity, but this dissociation is rapidly reduced to noise level for laser intensities that are only a few percent lower. Within a rather limited intensity interval (between $2.4 \times 10^{13}$ and 3.8 $\times 10^{13} \mathrm{~W} \mathrm{~cm}^{-2}$ ) they observe an increase of the toluene parent ion yield proportional to $I^{8.6}$, whereas for $780 \mathrm{~nm}$ one would again expect an ionization order of 6 .

Although our $\mathrm{C}_{7} \mathrm{H}_{8}^{+}$data points evidently follow a straight line with an effective slope of six, a description based on just a six-photon MPI process must be incomplete. For the wavelength and intensities used here, a considerable amount of ponderomotive (quiver) energy may be acquired by the electron after it has been freed from the molecular ionic core. In practical units, this ponderomotive energy $U_{p}$ is given (for $\lambda=800 \mathrm{~nm}$ ) by

$$
U_{p}(\mathrm{eV})=5.976 \times 10^{-14} I\left(\mathrm{~W} \mathrm{~cm}^{-2}\right),
$$

which, for the range of peak intensities used here, lies between 1.1 and $16.7 \mathrm{eV}$. The relative ac Stark shift between the ground state and the ionization energy can be considered to be equal to the ponderomotive energy. As a result, channel closing will occur (see Refs. 15 and 16 for similar effects in atoms), and a seven-photon process (not six) would be required (at least during part of the temporal evolution of the pulse) even for our lowest peak intensity, since $I E+U_{p}$ $=8.828+1.1 \mathrm{eV}=6.4 \hbar \omega$. Only for peak intensities below $7.9 \times 10^{12} \mathrm{~W} \mathrm{~cm}^{-2}$ could a six-photon process be active during the whole temporal evolution of the pulse. Channel closing would drastically change the order of the MPI process during the temporal evolution of the pulse: for our highest peak intensity, 17 photons would have to be absorbed to reach the ac Stark shifted ionization continuum! As will be demonstrated below, the slope of six observed in the present work is in fact an effective slope brought about by the combined operation of several different MPI processes with orders between 6 and 11 inclusive. In any case the slope of 8.6 observed in the measurements of DeWitt et al. within a rather narrow intensity range seems to contradict the present measurements. DeWitt et al. noted the unexpected character of their results and speculate on the possibility of abovethreshold ionization (ATI), i.e., absorption of more photons than the minimum number required for ionization. They immediately rule out this possibility, however, for three reasons: (1) their slope has a constant value (albeit over a narrow intensity range!), whereas they expect the absorption of additional photons for increasing intensities to increase the slope; (2) dissociation is "essentially absent" for benzene and toluene in their measurements, which indicates that no excess energy is absorbed by the molecule; and (3) no trend in the ionization order is observed for increasing molecular complexity $\quad$ (benzene $\rightarrow$ toluene $\rightarrow$ ethylbenzene $\rightarrow n$-propylbenzene), although they expect the density of states to increase for this series, which would favor ATI.

Because only one molecule is investigated here, we cannot say much about argument (3) of DeWitt et al. (the influence of molecular complexity on the order of the ionization process). However, inspection of the present results in view of arguments (1) and (2) is most instructive. Our results do seem to deviate slightly from a straight line in a concave way, i.e., before saturation sets in, the slope of the ionization 


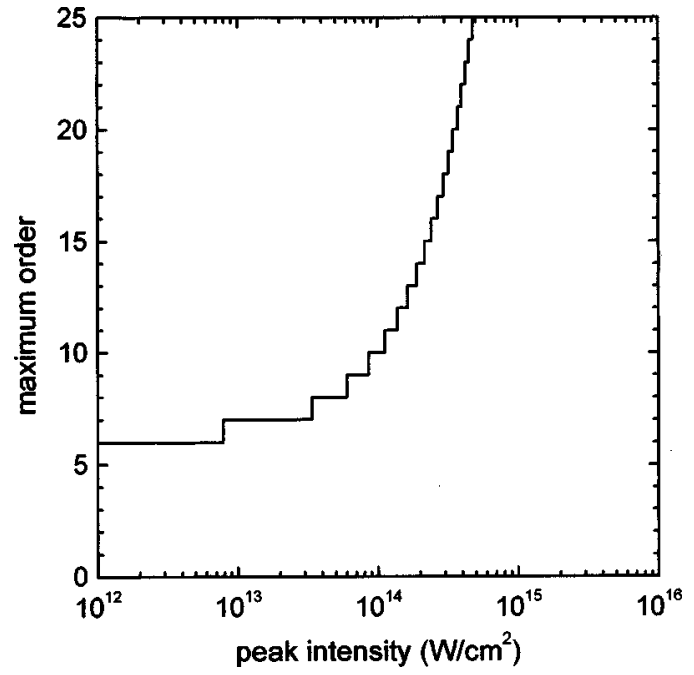

FIG. 3. Channel closing: highest order expected for an MPI process as a function of peak intensity for gaseous toluene and $800 \mathrm{~nm}$ radiation.

yield can be seen to increase somewhat for increasing intensities, although the average slope is six. Furthermore, we have a small but significant $\mathrm{C}_{7} \mathrm{H}_{7}^{+}$fragmentation signal for intensities beyond $\sim 6 \times 10^{13} \mathrm{~W} \mathrm{~cm}^{-2}$. To demonstrate how these observations can be explained as a result of the combined action of several MPI processes with different orders, it will be useful to write the spatio-temporal intensity distribution as $I(\mathbf{r}, t)=I_{0} G(\mathbf{r}) \exp \left(-\left(t / t_{0}\right)^{2}\right)$, where $\mathbf{r}=\mathbf{0}$ coincides with the center of the focus and $G(\mathbf{r}=\mathbf{0})=1$. For a given peak intensity $I_{0}(\mathbf{r})=I_{0} G(\mathbf{r})$, the $N$-photon process will be closed and the $(N+1)$-photon channel opened as soon as the intensity has reached the value $I\left(t_{N}\right)$ given by ${ }^{17}$

$$
I E+U_{p}=I E+5.976 \times 10^{-14} I\left(t_{N}\right)=N \hbar \omega,
$$

where the unit of energy is the eV and the intensity is measured in $\mathrm{W} \mathrm{cm}^{-2}$. In other words, the ionization probability per unit of time for a neutral molecule changes order during the temporal evolution of the pulse and is given on the rising edge of the pulse by

$$
\begin{array}{ll}
\sigma_{(6)}(I(t) / \hbar \omega)^{6} & \text { for } t \leqslant t_{6}, \\
\sigma_{(7)}(I(t) / \hbar \omega)^{7} & \text { for } t_{6}<t \leqslant t_{7}, \\
\sigma_{(8)}(I(t) / \hbar \omega)^{8} & \text { for } t_{7}<t \leqslant t_{8}, \\
\ldots & \text { etc., }
\end{array}
$$

where the highest order appearing depends on the peak intensity $I_{0}$, and by a similarly decreasing series of orders on the falling edge of the pulse. The quantities $\sigma_{(N)}$ are the generalized cross section for an $N$-photon MPI process, measured in units of $\mathrm{cm}^{2 N} \mathrm{~s}^{N-1}$. The ionization probability after the pulse is over is then given by

$$
\begin{aligned}
P\left(I_{0}\right)= & 1-\exp \left[-\left(\int_{-\infty}^{t_{6}} \sigma_{(6)}\left(\frac{I(t)}{\hbar \omega}\right)^{6} d t+\int_{t_{6}}^{t_{7}} \sigma_{(7)}\right.\right. \\
& \left.\left.\times\left(\frac{I(t)}{\hbar \omega}\right)^{7} d t+\int_{t_{7}}^{t_{8}} \sigma_{(8)}\left(\frac{I(t)}{\hbar \omega}\right)^{8} d t+\cdots\right)\right],
\end{aligned}
$$

with the actual number of different orders to be evaluated depending on the peak intensity $I_{0}$; in Fig. 3 this maximum order is plotted as a function of $I_{0}$ [cf. Eq. (6)]. In what follows, the generalized cross sections will be considered to have fixed values, i.e., they are taken to be intensityindependent. A calculation of these quantities for a molecule as complex as the 15-atom toluene molecule is prohibitively complicated; in any case it is way beyond the scope of the present work. Therefore, we will try to find values for them by comparing our calculations with the experimental data. To do so, we need to calculate the spatial integral of $P\left(I_{0}(\mathbf{r})\right)$ over the whole focal region; more specifically, the ion yield $Y\left(I_{0}\right)$ for a given value of the absolute peak intensity $I_{0}$ (i.e., the peak intensity in the center of the focus) is given by

$$
Y\left(I_{0}\right) \propto \iiint_{\text {all space }} P\left(I_{0}(\mathbf{r})\right) d \mathbf{r} .
$$

As an illustration to our multi-order model, Fig. 4 shows how the ionization probability per unit of time [as given in Eq. (7)] changes during the temporal evolution of the pulse for a number of different peak intensities. For peak intensities below $7.9 \times 10^{12} \mathrm{~W} \mathrm{~cm}^{-2}$ (where the 6-photon channel would close) the 6-photon process is active during the whole pulse [panels (a) and (b)]. For a peak intensity of 1.0 $\times 10^{13} \mathrm{~W} \mathrm{~cm}^{-2}$ [panel (c)], the 6-photon channel is closed in the center of the pulse, where now a 7-photon process is active; the 6-photon process is active in the wings only. The size of the discontinuous jump depends on the ratio between $\sigma_{(6)}$ and $\sigma_{(7)}$. For higher peak intensities [panels (d) and (e)], the activity of the 6-photon process is pushed more and more into the wings of the pulse, and the 7-photon process gradually starts dominating the ionization process. This process is closed in turn at an intensity of $3.4 \times 10^{13} \mathrm{~W} \mathrm{~cm}^{-2}$. For a peak intensity beyond this value [see panel (f)], the ionization process is composed of three different orders; a 6-photon process in the far wings of the pulse, a 7-photon process in the intermediate intensity regimes, and an 8-photon process in the center of the pulse, where the intensity is highest. In Fig. 5 the time-integrated ionization probability [Eq. (8)] is shown as a function of peak intensity (lower solid line, right ordinate axis). As a result of the discontinuities shown in Fig. 4, this probability has jumps at the channel-closing intensities as given by Eq. (6), although these jumps are somewhat smoothed due to the time integration. The volume integral according to Eq. (9) is given by the solid line passing through the parent ion data in Fig. 5. The agreement with our data is almost perfect. Values for the generalized cross sections obtained by fitting to our experimental data are given in Table I. Remarkably, we have to conclude that the inclusion of higher-order MPI processes still leads to an effective slope of 6. In our calculations, orders up to 11 have been included. Still, the curve through the data points in Fig. 5 never has a slope steeper than 7.9; this slope is found for a peak intensity of 9.4 $\times 10^{13} \mathrm{~W} \mathrm{~cm}^{-2}$. Perhaps the observation by DeWitt et al. ${ }^{6}$ of a slope of 8.6 in a rather narrow intensity range corresponds to such a sector in the parent ion yield curve. The considerations that made us choose the eleventh order as the highest 

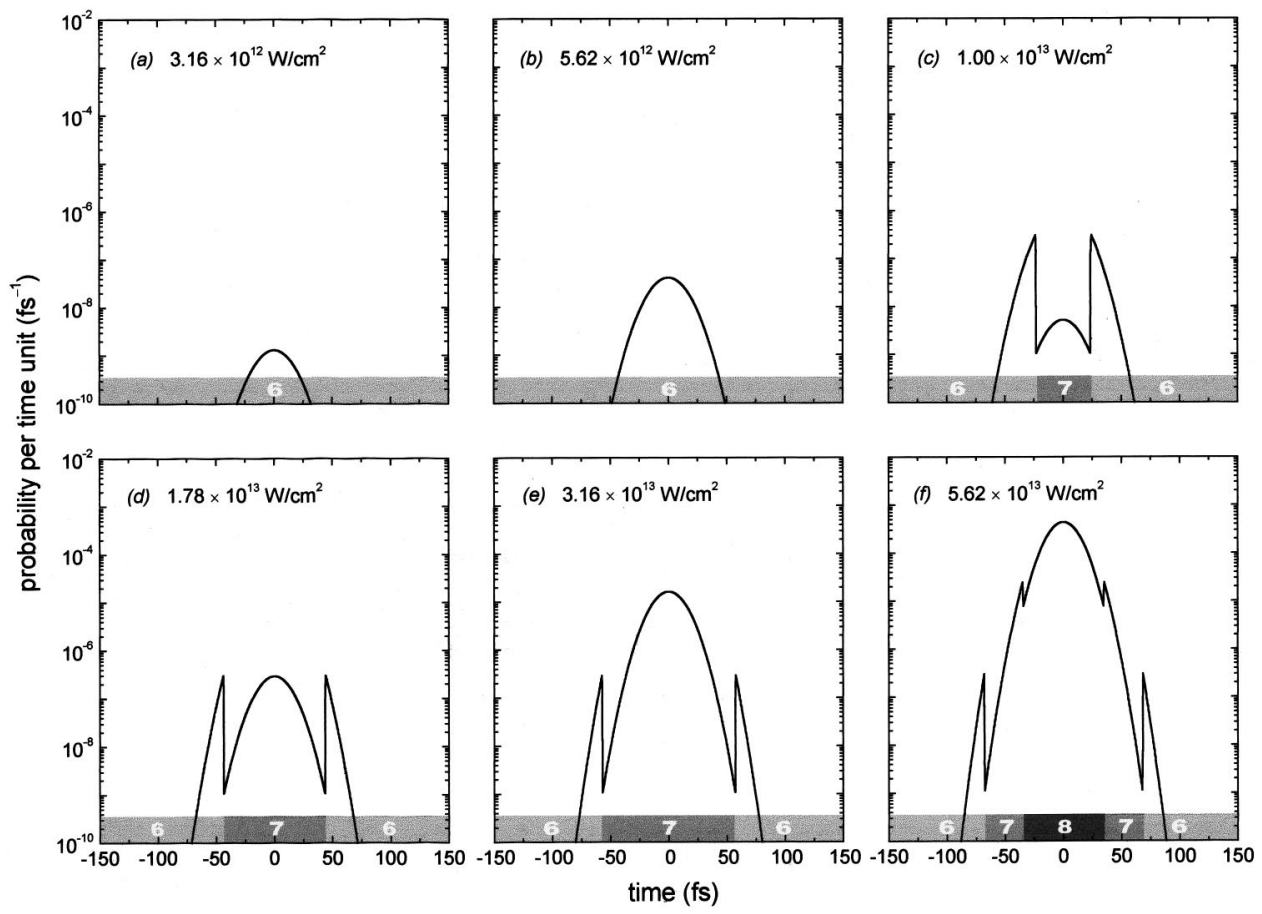

FIG. 4. Ionization probability per unit of time of a neutral gaseous toluene molecule for various peak intensities of $800-\mathrm{nm}, 80-\mathrm{fs}$, linearly polarized laser pulses. The discontinuities are due to channel closing. The order of the process is indicated in the strip at the bottom of each graph.

order deserve some discussion. Note that on a logarithmic scale the intensity intervals between two successive channelclosing intensities become smaller and smaller for increasing intensities (see Fig. 3). For our highest peak intensities, orders up to 17 could be expected in principle. We would then

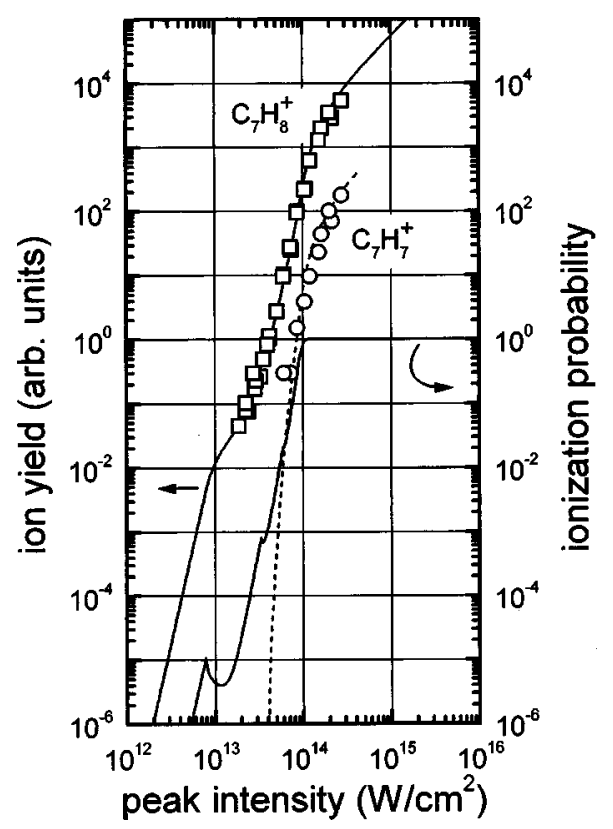

FIG. 5. Ion yields as a function of peak intensity for 800-nm, 80-fs, linearly polarized laser pulses irradiating gaseous toluene. Squares, parent ion, $\mathrm{C}_{7} \mathrm{H}_{8}^{+}$, circles, fragment ion, $\mathrm{C}_{7} \mathrm{H}_{7}^{+}$. The solid line passing through the parent ion data (left ordinate axis) was obtained by volume-integrating the ionization probability that results from the combined action of 6-, 7-, 8-, 9-, 10-, and 11-photon processes (lower solid curve, right ordinate axis). The jumps in the ionization probability curve are the result of channel closing (cf. Fig. 4). The dotted line shows the expected effective $\mathrm{C}_{7} \mathrm{H}_{7}^{+}$fragment ion yield on the assumption that the internal energy of the toluene cation equals the ponderomotive energy of the electron. have to fit values for a set of 12 different generalized cross sections. However, some arguments against including orders even higher than 11 can be given. First, the eleventh-order channel-closing intensity is at $1.4 \times 10^{14} \mathrm{~W} \mathrm{~cm}^{-2}$, less than a factor of 2 away from the highest intensity we have available, and the experimental data show that saturation already sets in for intensities around $\sim 10^{14} \mathrm{~W} \mathrm{~cm}^{-2}$. The more one penetrates into the saturation regime, the more the shape of the fitting curve is determined by volume effects, and, as a result, the shape of the fitting curve becomes increasingly immune to the value of very high-order $(N>10)$ generalized cross sections. In a self-consistent way, if the highest order included is $N_{\max }$, the values for $\sigma_{(6)}, \sigma_{(7)}, \ldots, \sigma_{\left(N_{\max }\right)}$ have to be chosen so that they describe an ionization process that saturates for a peak intensity that is lower than the $\left(N_{\max }\right)$-photon channel-closing intensity. The values given in Table I satisfy this condition. Second, the picture of an ever increasing MPI order for increasing intensities must break down at some point. After all, the Keldysh adiabaticity pa-

TABLE I. Generalized MPI cross sections $\sigma_{(N)}$ with orders $N$ between 6 and 11 of gaseous toluene under the influence of $800-\mathrm{nm}, 80-\mathrm{fs}$, linearly polarized laser pulses. The quantities $\Lambda_{N}$ defined as $\Lambda_{N}$ $=-(1 / N) \log _{10} \sigma_{(N)}$ reach a constant value for the highest orders $N$, which suggests that tunneling ionization is about to set in (Ref. 14) for intensities close to the saturation intensity (see text).

\begin{tabular}{ccc}
\hline \hline $\begin{array}{c}\text { Order } \\
N\end{array}$ & $\begin{array}{c}\text { Generalized MPI } \\
\text { cross section } \sigma_{(N)}\end{array}$ & $\Lambda_{N}=-\frac{1}{N} \log _{10} \sigma_{(N)}$ \\
\hline 6 & $3.1 \times 10^{-181} \mathrm{~cm}^{12} \mathrm{~s}^{5}$ & 30.0 \\
7 & $3.0 \times 10^{-215} \mathrm{~cm}^{14} \mathrm{~s}^{6}$ & 30.6 \\
8 & $6.4 \times 10^{-248} \mathrm{~cm}^{16} \mathrm{~s}^{7}$ & 30.9 \\
9 & $1.4 \times 10^{-280} \mathrm{~cm}^{18} \mathrm{~s}^{8}$ & 31.1 \\
10 & $5.4 \times 10^{-313} \mathrm{~cm}^{20} \mathrm{~s}^{9}$ & 31.2 \\
11 & $2.0 \times 10^{-344} \mathrm{~cm}^{22} \mathrm{~s}^{10}$ & 31.2 \\
\hline \hline
\end{tabular}


rameter starts to get smaller than unity at least during the central part of the pulse for peak intensities beyond the value $7.4 \times 10^{13} \mathrm{~W} \mathrm{~cm}^{-2}$ (the 9-photon channel closes at a slightly higher intensity, $8.6 \times 10^{13} \mathrm{~W} \mathrm{~cm}^{-2}$ ). Tunneling ionization and/or over-the-barrier processes could then at some point be expected to take over in the center of the pulses for highest intensities, with pure MPI processes still being active in the wings of the pulse. However, the value for unity for the Keldysh parameter should not be taken as "a strict demarcation line" but rather as a "characteristic region," as was stressed by Lambropoulos, ${ }^{14}$ who also showed that a constant value (independent of $N$ ) of the quantity $\Lambda_{N}$, defined as $\Lambda_{N}=-(1 / N) \log _{10} \sigma_{(N)}$, must be related to tunneling. Inspection of these quantities in our case (see Table I) show that they do have a tendency to approach some limit for increasing $N$, although they depend more strongly on $N$ than in the hydrogen case that is described in Ref. 14. In our model calculations we did not include tunneling and/or overthe-barrier processes; instead, for all peak intensities, we took the order of the process equal to the lowest possible, taking into account channel closing. Tunneling could be thought of as the simultaneous action of several processes with different orders, not just the lowest possible order as is the case in our model. Once inside the tunneling regime, the contributions of all these different processes become comparable. We demonstrated that the inclusion of MPI processes with (lowest possible) orders higher than 6 can still produce an effective slope of six, and that results of this procedure are in agreement with our experimental data. This justifies replacing the whole set of conceivable orders by merely the lowest-order-perturbation theory (LOPT) order, which in turn implies that tunneling is not a very dominant mechanism here. It must be noted that tunneling and/or over-the-barrier processes cannot be completely excluded as long as these field processes take place for intensities close to the saturation intensity, where the experimental ion yield is mainly determined by volume effects anyway. If tunneling and/or over-the-barrier processes contribute to the ionization process at all, their significance in the ionization process is confined to a narrow intensity regime, somewhere between $\sim 7.4 \times 10^{13} \mathrm{~W} \mathrm{~cm}^{-2}$ (where the adiabaticity parameter starts falling below unity) and $\sim 1 \times 10^{14} \mathrm{~W} \mathrm{~cm}^{-2}$ (the experimental saturation intensity).

As mentioned above, DeWitt et al. measured a negligible amount of fragmentation in their toluene experiments and used this observation as an argument against absorption of excess photons. Now in our experiments, we do observe a clear signal from the fragmentation product $\mathrm{C}_{7} \mathrm{H}_{7}^{+}$(see Figs. 1 and 2) which becomes detectable for intensities above $\sim 6 \times 10^{13} \mathrm{~W} \mathrm{~cm}^{-2}$. We will now demonstrate how our fragmentation data support our multiple-order MPI model with orders up to 11 inclusive. Fragmentation of the excited toluene cation is known ${ }^{18}$ to produce two isomers of $\mathrm{C}_{7} \mathrm{H}_{7}^{+}$, tropylium and benzylium. Since our TOF method merely detects mass/charge ratios, this fragmentation can be symbolically written as

$$
\mathrm{C}_{7} \mathrm{H}_{8}^{+}\left(E_{\text {int }}\right) \stackrel{k\left(E_{\text {int }}\right)}{\longrightarrow} \mathrm{C}_{7} \mathrm{H}_{7}^{+}+\mathrm{H} .
$$

The lowest energy secondary fragmentation process is the breakdown of the formed $\mathrm{C}_{7} \mathrm{H}_{7}^{+}$into $\mathrm{C}_{5} \mathrm{H}_{5}^{+}$and $\mathrm{C}_{2} \mathrm{H}_{2}{ }^{18}$ The appearance energy of the $\mathrm{C}_{5} \mathrm{H}_{5}^{+}$fragment starting from the neutral toluene molecule is $14.55 \mathrm{eV}$, but for detection times of the order of $10 \mu \mathrm{s}$, it appears only beyond $\sim 16 \mathrm{eV}{ }^{18} \mathrm{In}$ our experiments, the $\mathrm{C}_{5} \mathrm{H}_{5}^{+}$fragment is not observed; this fact is consistent with our multiple-order MPI model, as will be shown below. Rate-energy functions $k\left(E_{\text {int }}\right)$ for the unimolecular decay reaction (10) are given in Refs. 18 and 19, where the internal energy dependence of the fragmentation was investigated by photoelectron-photoion coincidence spectroscopy (PEPICO) starting from neutral toluene. In Ref. 18 , a RRKM expression for $k\left(E_{\text {int }}\right)$ was fitted to the breakdown curve of $\mathrm{C}_{7} \mathrm{H}_{8}^{+}$close to the onset of reaction (10) in the energy range $11.2 \mathrm{eV} \leqslant \mathrm{IE}+E_{\mathrm{int}} \leqslant 12.1 \mathrm{eV}$ (IE being the ionization energy of the toluene molecule). The authors of Ref. 18 conclude that their results can only be explained by two competitive unimolecular decay processes, and identify these processes as the formation of tropylium and benzylium. For $I E+E_{\text {int }} \lesssim 12.1 \mathrm{eV}$, the formation of tropylium dominates. For higher internal energies, the fitted RRKM curves predict that the faster but more endothermal benzylium formation is favored. For $I E+E_{\text {int }} \approx 12 \mathrm{eV}$, the total rate is about $10^{5} \mathrm{~s}^{-1}$, and extrapolating their RRKM results, these authors predict the rate to rise to above $10^{8} \mathrm{~s}^{-1}$ for $I E+E_{\mathrm{int}} \approx 13.7 \mathrm{eV}$. The authors of Ref. 19 also used the PEPICO method, but come to the conclusion that the rate reaches a constant value of $k$ $=(1.5 \pm 0.4) \times 10^{5} \mathrm{~s}^{-1}$ for excitation energies of the toluene parent ion exceeding $3.7 \mathrm{eV}$. In the present experiment, no $\mathrm{C}_{7} \mathrm{H}_{7}^{+}$signal is observed for peak intensities below $\sim 6$ $\times 10^{13} \mathrm{~W} \mathrm{~cm}^{-2}$, and beyond this threshold intensity the yield is about $3 \%$ of the parent ion yield, this ratio being almost independent of the peak intensity. For a correct interpretation of this fragmentation yield, the so-called kinetic shift must be taken into account; since one is dealing here with fragmentation times that are comparable to the time between the creation of an ion and its detection (the time-of-flight), fragmentation processes take place during the flight of the ions through the TOF spectrometer. The time-of-flight of the $\mathrm{C}_{7} \mathrm{H}_{7}^{+}$fragment will depend on (i) which sector of our TOF spectrometer the fragmentation takes place in and (ii) what amount of extra kinetic energy is gained by the $\mathrm{C}_{7} \mathrm{H}_{7}^{+}$fragment as a result of the fragmentation. In order to clarify our fragmentation data, we simulated our TOF spectra by making trajectory calculations for toluene ions that undergo a fragmentation process governed by a single exponential decay in time. As described in Ref. 18, the amount of translational energy that is deposited in the $\mathrm{C}_{7} \mathrm{H}_{7}^{+}$fragment as a result of the fragmentation reaction (10) is very small; at most $10 \%$ of the excess energy in the parent ion is converted into translational energy of the products, and the light hydrogen atom will carry away almost $99 \%$ of this energy. As a result, the translational energy deposited in the $\mathrm{C}_{7} \mathrm{H}_{7}^{+}$fragment is less than the thermal kinetic energy of the parent ion it is created from, and it is neglected in our simulations. Indeed, the experimental widths of the peaks at $M=91$ and $M=92$ are not significantly different, and they agree very well with our simulations, assuming an initial $298 \mathrm{~K}$ thermal velocity distribution of the neutral toluene molecules. Thus, 
for a fragmentation process taking place in the field-free drift sector of our spectrometer (where the ions spend most of their time), the difference in speed between the $\mathrm{C}_{7} \mathrm{H}_{7}^{+}$fragment produced and the parent ion it was created from is negligible, and the time-of-flight of this fragment will therefore be identical to that of a parent ion, i.e., as if the parent ion did not decay at all. Our simulations show that the observed ratio of $3 \%$ between the heights of the peaks at $M$ $=91$ and $M=92$ corresponds to a fragmentation rate $k$ $=0.4 \times 10^{5} \mathrm{~s}^{-1}$, but that in spite of this seemingly modest amount of fragmentation the real fragment ratio is much higher; in fact, during the total flight time of $t_{92}=12.86 \mu \mathrm{s}$ for the parent ion as many as $\sim 40 \%\left(=1-e^{k \cdot t_{92}}\right)$ of the parent ions undergo dissociation.

In Fig. 5 an (apparent) fragment ion yield curve (dashed line) is shown that was numerically calculated using the rates given in Ref. 19 after dividing these by a factor of 4.5 . The calculation was done as follows. To make use of known rate-energy curves, one needs to know the amount of internal energy deposited by the photoionization process into the toluene parent ion. An obvious way to deposit energy in the ion is by recollision of the electron just after it is freed from the ionic core and starts its quiver motion. Such a recollision model was developed by Corkum et al. ${ }^{20}$ to explain higherharmonic generation. For a monchromatic wave and an atomic ionic core (i.e., a core of negligible spatial extent), the maximum return energy of the electron is $\sim 3.17$ times the ponderomotive energy, but already in the first few cycles the electron can return to the vicinity of the ion with a considerable amount of kinetic energy. In the case of molecules, the ionic core that the electron returns to is still in the nuclear configuration of the neutral molecule in its ground state. To explain the fragmentation of the toluene cations, it will now be assumed that recollision of the electron and the core will bring a certain amount of excess energy into the system, and this amount is assumed to equal the ponderomotive energy $U_{p}$ as given in Eq. (5), where we take the intensity that prevailed at the moment of ionization to calculate $U_{p}$. Then, after the laser pulse is over, this excess energy is assumed to be rapidly converted into vibrational energy of the unperturbed toluene cation in its ground electronic state, so that finally the ponderomotive energy at the time of photoionization becomes available to the toluene cation as internal energy. For each peak intensity, we numerically determined the internal energy distribution for the created ensemble of toluene cations, taking the volume integral into account. Finally, using trajectory calculations, we have determined the fraction of cations that will end up in the peak corresponding to $\mathrm{C}_{7} \mathrm{H}_{7}^{+}$after their flight through our TOF spectrometer. Figure 5 shows that the agreement between the fragmentation yield curve thus calculated and the experiment is very satisfactory, including the detection threshold at $\sim 6 \times 10^{13} \mathrm{~W} \mathrm{~cm}^{-2}$. At this point we will consider the absence of the $\mathrm{C}_{5} \mathrm{H}_{5}^{+}$fragment again. For a detection time of the order of $10 \mu$ s, the appearance energy of this fragment starting from the neutral toluene molecule is $\sim 16 \mathrm{eV} .^{18}$ This amount of internal energy could just be reached with an 11-photon absorption process. However, as a result of saturation, few molecules survive the rising edge of the pulse to get ionized with so many photons.
Our simulations show that even for the highest peak intensity used here, the fraction of molecules with an internal energy of $15.75 \mathrm{eV}$ or higher is of the order of $10^{-6}$. Therefore, the absence of the $\mathrm{C}_{5} \mathrm{H}_{5}^{+}$fragment confirms our model, in which saturation effectively suppresses the occurrence of MPI processes with orders beyond 11 . If tunneling were very dominant, the tenth and lower order processes would be accompanied by non-negligible contributions from eleven-photon processes, but the absence of the $\mathrm{C}_{5} \mathrm{H}_{5}^{+}$fragment leaves no doubt that 11-photon MPI must be of negligible importance. Again, it is seen that the LOPT-MPI picture describes our data sufficiently well. Unfortunately, one can only roughly estimate the amount of excess energy that can be deposited into the ion as a result of recollisions, but the estimation of this excess energy by the ponderomotive energy gives a satisfying reproduction of the measured fragmentation data, even though this estimation is rather on the low side in relation to the $U_{p} 3.17$ maximum recollision energy found for the atomic case. The nonmaximum amount of recollision kinetic energy can perhaps be explained by the fact that the toluene ionic core is greater in extent than an atomic ion, so that collisions between an accelerating quasi-free electron and the ionic core before the electron ever managed to reach its first turning point are conceivable, which could reduce the amount of kinetic energy the electron can acquire from the field. In any case the absence of the $\mathrm{C}_{5} \mathrm{H}_{5}^{+}$fragment clearly proves that the amount of internal energy deposited into the ion must be limited. In particular, this fragment is also absent for our highest peak intensity of $2.8 \times 10^{14} \mathrm{~W} \mathrm{~cm}^{-2}$, in which case a ponderomotive energy of more than $16 \mathrm{eV}$ could be acquired by the electron. In our model, it is the intensity at the moment of ionization that is taken to calculate the ponderomotive energy, not the peak intensity. Our maximum peak intensity of $2.8 \times 10^{14} \mathrm{~W} \mathrm{~cm}^{-2}$ brings us well into the saturation regime, where there is a large difference between the peak intensity and the highest intensity on the rising edge of the pulse that still leads to non-negligible amounts of ionization. The lack of $\mathrm{C}_{5} \mathrm{H}_{5}^{+}$even for the highest intensities supports our model, in which the electron's kinetic energy is calculated with the intensity that prevailed at the moment of ionization, and also suggests that the interaction between the electron and the ionic core takes place within a few optical cycles after the ionization took place. Successive channel closing and LOPT-MPI processes of orders up to 11 inclusive are all taken into account in our calculations of the internal energy distribution, and the resulting fragmentation is in good agreement (within a factor of the order of unity) with the rate-energy curve of Ref. 19. We therefore conclude that the photoionization of the toluene molecule using linearly polarized, $800 \mathrm{~nm}, 80 \mathrm{fs}$ laser pulses is strongly dominated by LOPT-MPI processes, taking successive channel closing into account.

To shed more light on the ionization mechanism, we made polarization-dependent measurements. In Fig. 6, the parent ion and $\mathrm{C}_{7} \mathrm{H}_{7}^{+}$yields are shown as a function of intensity for circularly polarized light normalized to the same (arbitrary) scale as the ion yields for linearly polarized light. The data in Fig. 6 were taken under the same conditions as the data of Fig. 5; all measurements involved one and the 


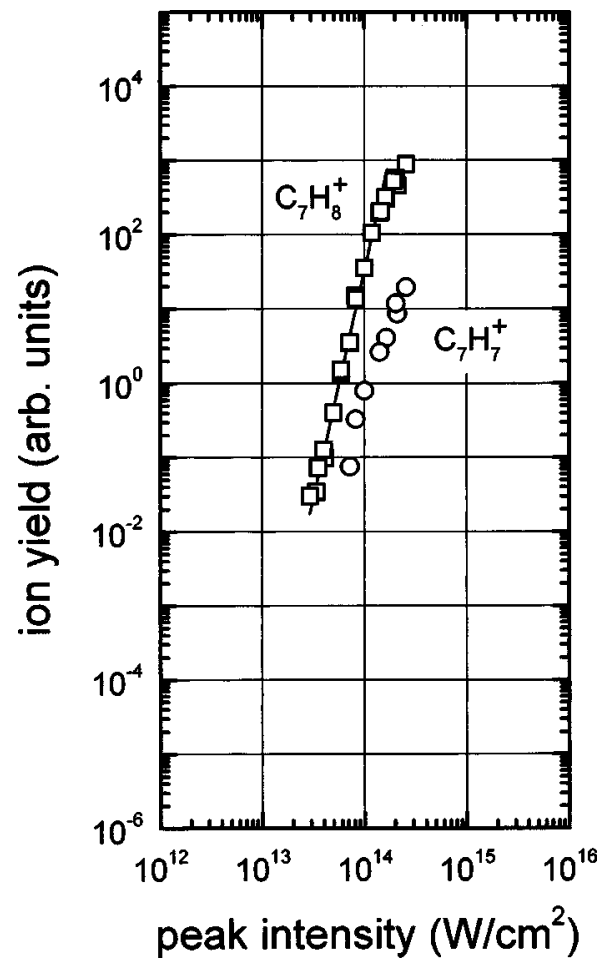

FIG. 6. Ion yields as a function of peak intensity for 800-nm, 80-fs, circularly polarized laser pulses irradiating gaseous toluene. Squares, parent ion, $\mathrm{C}_{7} \mathrm{H}_{8}^{+}$; circles, fragment ion, $\mathrm{C}_{7} \mathrm{H}_{7}^{+}$.

same quarter-wave plate in the beam. To change the polarization from linear to circular, the fast axis of this quarterwave plate was moved from $0^{\circ}$ (i.e., parallel to the linear polarization direction of the laser) to $45^{\circ}$. For circular polarization, the saturation intensity is somewhat higher than in the linear polarization case, $\sim 1.4 \times 10^{14} \mathrm{~W} \mathrm{~cm}^{-2}$. This value was determined as the peak intensity for which the parent ion yield is as high as it is for the saturation intensity using linear polarization. Due to the higher saturation intensity, fewer data points can be recorded in the saturation regime, which impedes accurate determination of the saturation intensity by fitting a curve to the data points. In the circular polarization case, an effective $I^{6}$ dependence is again found before saturation is reached; fitting a straight line through the nonsaturated part of this curve gives a slope of $6.14 \pm 0.17$. On the basis of this similarity between the curves, we made one other measurement (see Fig. 7), in which we kept the intensity fixed at the value $8.5 \times 10^{13} \mathrm{~W} \mathrm{~cm}^{-2}$, and recorded parent ion yields as a function of the ellipticity of the light by varying the angle $\theta$ between the fast axis of the quarter-wave plate and the direction of the linear polarization of the laser. With $Y_{\text {lin }}$ and $Y_{\text {circ }}$ denoting the parent ion yields for, respectively, linear polarization $\left(\theta=0^{\circ}\right)$ and circular polarization $\left(\theta=45^{\circ}\right)$ these data are well described by

$$
Y(\theta)=Y_{\text {circ }}+\left(Y_{\text {lin }}-Y_{\text {circ }}\right) \cos ^{2}(2 \theta),
$$

and from a fit to the data we find $\left(Y_{\text {lin }} / Y_{\text {circ }}\right)^{1 / 6}=1.4$, which confirms the ratio between the saturation intensities for linear and circular polarization.

As a consequence of the higher saturation intensity for circular polarization, ionization processes can now take place

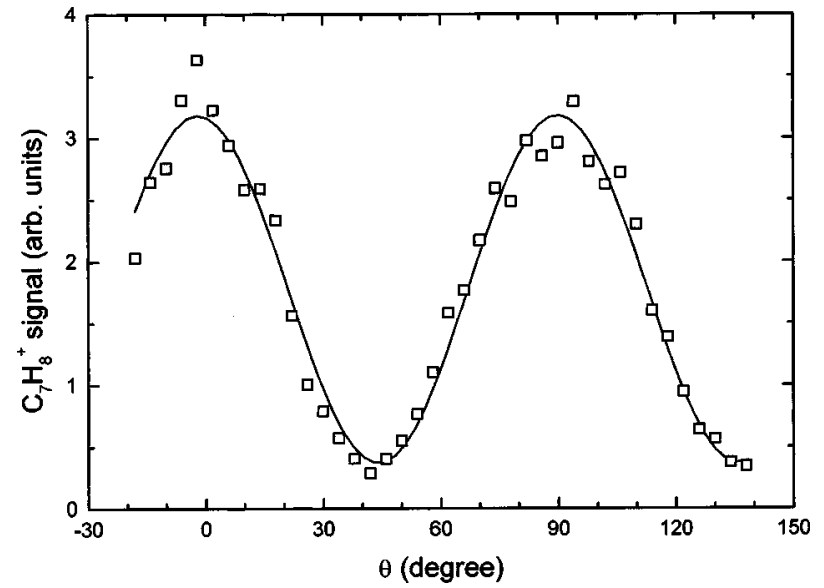

FIG. 7. Parent ion yield as a function of the ellipticity for $800 \mathrm{~nm}, 80 \mathrm{fs}$, laser pulses irradiating gaseous toluene, for a fixed peak intensity of 8.5 $\times 10^{13} \mathrm{~W} \mathrm{~cm}^{-2}$, measured by varying the angle $\theta$ between the fast axis of the quarter-wave plate and the polarization direction of incoming linearly polarized light (see text).

at intensities for which in the linear case there were no longer any neutral molecules left. This implies that, although field-ionization mechanisms could be excluded for the case of linear polarization, the ionization mechanism will now have to be reconsidered in view of the higher intensities the molecules can be exposed to with circular polarization. The 11-photon channel closes at $1.38 \times 10^{14} \mathrm{~W} \mathrm{~cm}^{-2}$, a value close to the saturation intensity. Within the framework of our multiple-order model, one could now include the twelfth, thirteenth, etc., orders and try to find values for the corresponding generalized cross sections in a consistent way as described before. Knowing that the eleventh order was already needed for the case of linear polarization, it is immediately clear that at least the twelfth order would now have to be included. This 12-photon channel would start to be active from the 11-photon channel-closing intensity of 1.38 $\times 10^{14} \mathrm{~W} \mathrm{~cm}^{-2}$, which is close to the experimental saturation intensity. Because a substantial fraction of the molecules is always ionized for intensities beyond the saturation intensity, a non-negligible amount of toluene cations would then be formed with internal energies between $8.2 \mathrm{eV}(11 \hbar \omega-I E)$ and $9.8 \mathrm{eV}(12 \hbar \omega-I E)$. Such internal energies would be more than enough to see the secondary fragment $\mathrm{C}_{5} \mathrm{H}_{5}^{+}$, but this species is not observed in our experiment! Within the scenario of a recolliding electron bringing an excess energy of the order of the ponderomotive energy into the cation, the lack of the secondary fragment, $\mathrm{C}_{5} \mathrm{H}_{5}^{+}$, for the circular polarization case in spite of the higher intensity can be made plausible by the fact that the semiclassical electron trajectories are now different. For circular polarization, the trajectory of a free electron is like an outgoing spiral, and it never really returns to the position it started at, as it could do in the linear polarization case. This may hinder the energy deposition mechanism.

As already mentioned, for a Keldysh adiabaticity parameter of the order of unity, as is the case in the present work, both MPI and field-ionization mechanisms could be anticipated. For (poly)aromatic molecules the simultaneous occur- 
rence of the two mechanisms has been deduced from the structure of the photoelectron spectra. ${ }^{5}$ The reduction in the ion yield observed for circular polarization could be made plausible for both mechanisms. For the case of MPI using circular polarization, the selection rules for the magnetic quantum number $M_{j}$ ( $J$ being the total angular momentum of the particle to be ionized) prescribe that $\Delta M_{J}=+1$ or -1 (the sign depending on the helicity of the polarization, i.e., left-or right-hand) for each intermediate absorption step. The reduction may then be ascribed to the reduced number of states that is addressable under this condition in comparison with the linear case, for which the less stringent selection rule $\Delta M_{J}=0, \pm 1$ applies. In a perturbative, nonresonant picture, the generalized cross section is proportional to a $(K$ $-1)$-fold summation of probability amplitudes squared $(K$ being the lowest number of photons required for ionization), with each summation extending over all allowed states (Ref. 13 describes the atomic case). Clearly, this sum will be smaller as the selection rules become more stringent. Therefore, insofar as this atomic MPI picture holds for the molecular case, a reduction can be rationalized (see Ref. 21 for a similar effect in $\mathrm{He}$ ). However, a reduction could also be compatible with a field-ionization mechanism. It must be noted that, for equal intensities, the peak electric field strength for $\varepsilon=0$ (linear polarization) is a factor of $\sqrt{2}$ larger than the peak electric field strength for $\varepsilon=1$ (circular polarization). Experiments with $\mathrm{Ne}$ and Xe using $1053 \mathrm{~nm}, 1.9 \mathrm{ps}$ pulses in the intensity range $10^{13}-10^{15} \mathrm{~W} \mathrm{~cm}^{-2}$ (Ref. 22) have shown that singly charged ions of these noble gases are produced by a tunneling ionization mechanism, and that a change of the polarization from linear to circular for a fixed intensity leads to a significant decrease of the ion signal. For the whole intensity range studied in Ref. 22 , however, the original ion signal is regained if the change in polarization from linear to circular is accompanied by an increase of the intensity by a factor of 1.5 (for $\mathrm{Ne}$ ) or 1.7 (for $\mathrm{Xe}$ ), and these factors are shown to be compatible with ADK tunneling theory. For an over-the-barrier process for which the frequency of the light field is irrelevant (see Ref. 22), the ionization probability depends only on the field strength, so that a factor of exactly two would be expected in that case. (Note that in order to have equal electric field strengths for both polarizations, the intensity used in the circular polarization case needs to be two times higher than in the linear polarization case.) For molecules, the situation must be more complicated, due to orientational effects. For small molecules, for instance, it has been shown that the ionization probability for linear polarization may depend on the orientation of the molecule in the field. ${ }^{23-25}$ Such orientational effects play a negligible role here as is evident from the following argumentation that is based on the assumption that the light field does not align the toluene molecules, so that their orientation in space can be taken random and frozen during the whole temporal evolution of the pulse. If a linearly polarized field is then applied, there will always be a limited fraction of the toluene molecules present within our detection volume for which the orientation with respect to the electric field happens to be optimal for ionization. If a circularly polarized field (with the same peak field strength) is applied, a larger fraction of the molecules can be addressed, due to the constantly changing orientation of the electric field vector in time, so that ion signal is expected to increase. Apparently orientational effects, if present at all, are completely counteracted by the reduced overall ionization efficiency of the circularly polarized light.

\section{CONCLUSIONS}

The results of the present experiment were obtained in an attempt to elucidate the ionization and fragmentation mechanism of a medium-sized organic molecule. In general, this mechanism can have both MPI and field aspects. We have shown that the photoionization of the toluene molecule in the gas phase under the influence of $80-\mathrm{fs}, 800-\mathrm{nm}$, linearly polarized laser pulses with peak intensities up to 2.8 $\times 10^{14} \mathrm{~W} \mathrm{~cm}^{-2}$ is well described by a multiple lowest perturbation order MPI model where orders up to 11 are included and channel closing is taken into account. Tunneling and/or over-the-barrier mechanisms are shown to be of minor importance. This ionization mechanism is in agreement with the observed effective sixth-order dependence of the parent ion yield on the intensity. An electron recollision model in which an excess energy of the order of the ponderomotive energy is brought into the toluene cation is shown to be in good agreement with existing literature data on the internal energy dependence of the fragmentation of the toluene cation into $\mathrm{C}_{7} \mathrm{H}_{7}^{+}$and $\mathrm{H}$. The general reduction of the ion yield that is observed when circular polarization is applied and the lack of the secondary fragmentation product, $\mathrm{C}_{5} \mathrm{H}_{5}^{+}$, can be explained by the reduced recollision probability of the electron in that case. It has also been demonstrated that a low amount of non-linear distortion of the beam, as expressed by the $B$-integral, is of paramount importance for molecular photoionization and photofragmentation experiments: higher charge states and extensive fragmentation are easily observed if this $B$-integral grows above generally accepted upper limits.

\section{ACKNOWLEDGMENTS}

C.J.G.J.U. gratefully acknowledges the award of stipends from the Alexander von Humboldt-Stiftung and the Max-Planck-Gesellschaft. B.W. is obliged to the MaxPlanck-Institut für Quantenoptik for the possibility of participating in this work. With pleasure the authors acknowledge fruitful discussions with P. Lambropoulos. They are grateful to Th. Schikarski for many useful discussions and for his help in setting up the electronics. They thank H. Schröder and C. R. Gebhardt for stimulating discussions. They are obliged to W. E. Schmid for making the Wiley-McLaren TOF spectrometer available to them. They also acknowledge the technical assistance of W. Ritt and A. Steyer.

${ }^{1}$ K. W. D. Ledingham, D. J. Smith, R. P. Singhal, T. McCanny, P. Graham, H. S. Kilic, W. X. Peng, S. L. Wang, A. J. Langley, P. F. Taday, and C. Kosmidis, J. Phys. Chem. A 103, 2952 (1999).

${ }^{2}$ D. J. Smith, K. W. D. Ledingham, R. P. Singhal, H. S. Kilic, T. McCanny, A. J. Langley, P. F. Taday, and C. Kosmidis, Rapid Commun. Mass Spectrom. 12, 813 (1998).

${ }^{3}$ K. W. D. Ledingham, R. P. Singhal, D. J. Smith, T. McCanny, P. Graham, 
H. S. Kilic, W. X. Peng, S. L. Wang, A. J. Langley, P. F. Taday, and C. Kosmidis, J. Phys. Chem. A 102, 3002 (1998).

${ }^{4}$ M. Castillejo, S. Couris, E. Koudoumas, and M. Martín, Chem. Phys. Lett. 308, 373 (1999).

${ }^{5}$ M. J. DeWitt and R. J. Levis, Phys. Rev. Lett. 81, 5101 (1998).

${ }^{6}$ M. J. DeWitt, D. W. Peters, and R. J. Levis, Chem. Phys. 218, 211 (1997).

${ }^{7}$ M. J. DeWitt and R. J. Levis, J. Chem. Phys. 108, 7045 (1998).

${ }^{8}$ A. E. Siegman, Lasers (University Science Books, Mill Valley, California, 1986).

${ }^{9}$ L. V. Keldysh, Zh. Eksp. Teor. Fiz. 47, 1945 (1964); [Sov. Phys. JETP 20, 1307 (1965)].

${ }^{10}$ W. C. Wiley and I. H. McLaren, Rev. Sci. Instrum. 26, 1150 (1955).

${ }^{11}$ A. Brodeur and S. L. Chin, Phys. Rev. Lett. 80, 4406 (1998).

${ }^{12}$ S. G. Lias, Ionization Energy Evaluation, in NIST Chemistry WebBook, NIST Standard Reference Database Number 69, edited by W. G. Mallard and P. J. Linstrom, November 1998, National Institute of Standards and Technology, Gaithersburg, MD, 20899 (http://webbook.nist.gov).

${ }^{13}$ P. Lambropoulos, Comments At. Mol. Phys. 20, 199 (1987).

${ }^{14}$ P. Lambropoulos, Phys. Rev. Lett. 55, 2141 (1985).
${ }^{15}$ V. Schyja, T. Lang, and H. Helm, Phys. Rev. A 57, 3692 (1998).

${ }^{16}$ J. G. Story, D. I. Duncan, and T. F. Gallagher, Phys. Rev. A 49, 3875 (1993).

${ }^{17}$ For clarity, sometimes the explicit notation of the spatial dependence of the intensity is omitted.

${ }^{18}$ R. Bombach, J. Dannacher, and J.-P. Stadelmann, J. Am. Chem. Soc. 105, 4205 (1983)

${ }^{19}$ A. V. Golovin, M. E. Akopyan, and Yu. L. Sergeev, Khim. Fiz. 1983, 379 (1983) [Sov. J. Chem. Phys. 2, 632 (1985)].

${ }^{20}$ P. B. Corkum, Phys. Rev. Lett. 71, 1994 (1993).

${ }^{21}$ C. J. G. J. Uiterwaal, D. Xenakis, and D. Charalambidis, Z. Phys. D: At., Mol. Clusters 38, 309 (1996).

${ }^{22}$ S. Augst and D. D. Meyerhofer, Laser Phys. 4, 1155 (1994).

${ }^{23}$ A. D. Bandrauk and J. Ruel, Phys. Rev. A 59, 2153 (1999).

${ }^{24}$ M. Schmidt, S. Dobosz, P. Meynadier, P. D'Oliveira, and D. Normand, Phys. Rev. A 60, 4706 (1999).

${ }^{25}$ J. H. Posthumus, J. Plumridge, L. J. Frasinski, K. Codling, A. J. Langley, and P. F. Taday, J. Phys. B 31, L985 (1998). 